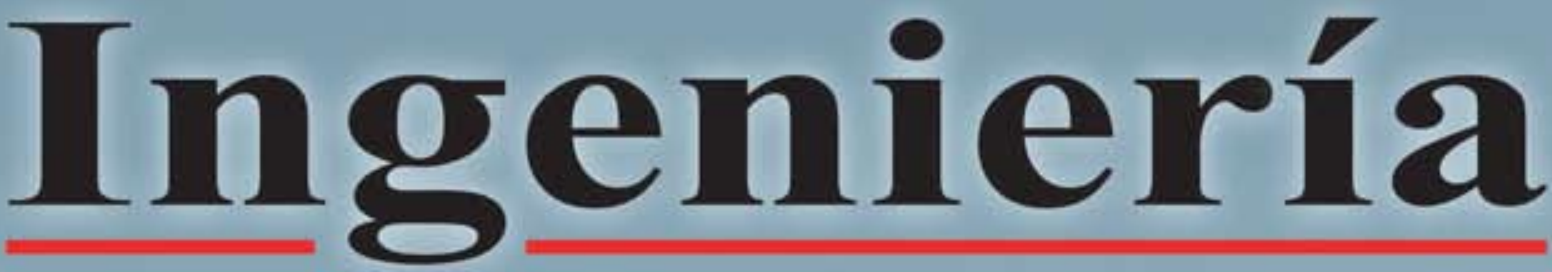

ENERO/DICIEMBRE 2004 - VOLUMEN 14 - N¹ y 2

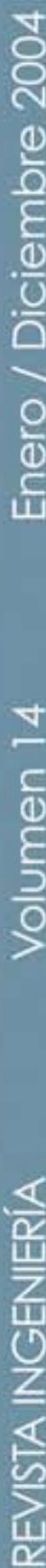
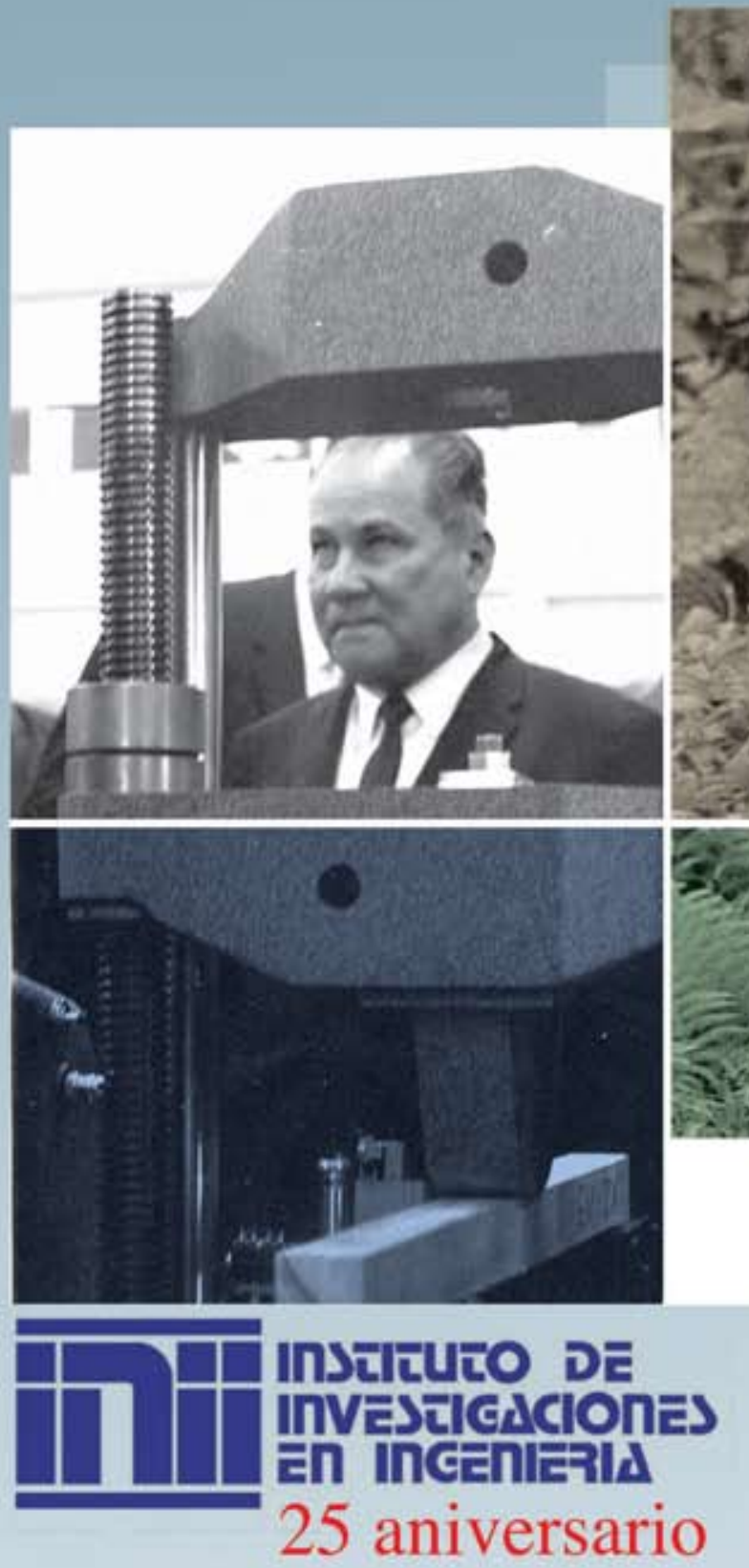

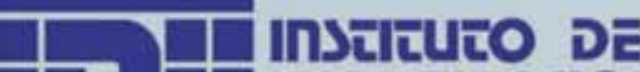
T W Investcscines हn Incenlei|d 25 aniversario

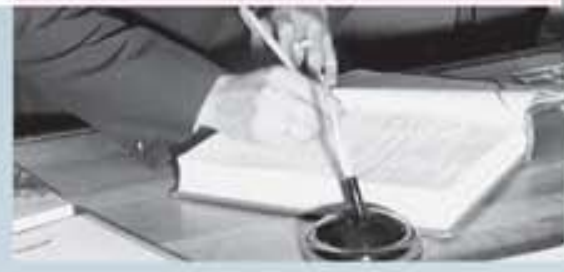




\title{
CONTROL NO LINEAL DE UN TCSC PARA MEJORAR LA ESTABILIDAD DE UN SISTEMA DE POTENCIA
}

\author{
Eddie A. Araya Padilla
}

\begin{abstract}
Resumen
Este artículo presenta el diseño del sistema de control de un compensador en serie controlado por tiristores o TCSC (TCSC: Thyristor-Controlled Series Compensation), basado en la combinación de la técnica de linealización entradasalida y la teoría del control óptimo lineal.

El desempeño del controlador propuesto, es verificado mediante la simulación de dos sistemas de potencia vinculados a través de una línea de transmisión. Los resultados obtenidos muestran que la estabilidad transitoria del sistema, presenta una mejora significativa al compararse con diseños convencionales de sistemas en serie de compensación fija.
\end{abstract}

Palabras clave: compensador en serie, control óptimo lineal, linealización entrada-salida

\begin{abstract}
This article presents the design of the control system of a compensator in series which is controlled by transistors or TCSC (Thyristor-Controlled Series Compensation). It is based on the combination of the input-output linearization technique and the theory for optimum lineal control.

The performance of the proposed controller is verified by the simulation of two power systems connected by a transmission line. The results show that the transitory stability of the system improves significantly, compared to the conventional designs for systems in series with fixed compensation.
\end{abstract}

Keywords: compensator in series, optimun lineal control, input-output linearization.

Recibido 29-VII-04 • Aceptado 14-X-04

\section{INTRODUCCIÓN}

Uno de los problemas de control más importantes en un sistema de potencia es la estabilización de un generador sincrónico. El generador, usualmente, está equipado con un regulador automático de tensión (AVR: automatic voltage regulator), para mantener la tensión terminal en un valor preestablecido, y un estabilizador de sistemas de potencia (PSS: power system stabilizer) para proporcionar un par de amortiguamiento y sincronización (Kundur, 1994). Sin embargo, el diseño convencional AVR/PSS tiene dos deficiencias (Wang et al., 1997): i) el diseño de ambos controles es desarrollado en forma independiente, disminuyendo la eficacia del conjunto; y ii) los controladores son diseñados mediante técnicas lineales, los cuales depende de las condiciones operativas, disminuyendo el rendimiento de los dispositivos cuando las condiciones varían.

Para considerar las no linealidades inherentes en los sistemas de potencia, debe orientarse el enfoque de diseño hacia herramientas no lineales.

Los enfoques no lineales, ya han sido utilizados en el pasado con resultados prometedores (Akhrif, Okou, Dessaint, Champagne, 1999; 
Cao, Wu, Jiang \& Cheng, 1998; Chapman, Ilic \& King, 1993; Gao, Jiang, Cheng, Chen, Malik \& Hope, 1994; Lu \& Sun, 1989; Lu, Sun, Xu \& Mochizuki, 1996; Wang, Guo \& Hill, 1997; Zhu, Zhou \& Wang, 1997).

Lu \& Sun (1989) utilizan la teoría geométrica diferencial para representar al modelo matemático en forma de un sistema afín no lineal, apto para el diseño del sistema de control. La virtud de este enfoque es que la ley de control es óptima, descentralizada e independiente de los parámetros de la red del sistema de potencia. Chapman et al. (1993) presentan una combinación de la técnica de linealización retroalimentada y la observación desacoplada del espacio de estado. La ventaja de esta propuesta es que permite el uso de señales medidas localmente y cuyo desempeño es consistente con respecto a cambios topológicos de la red, condiciones de carga y despachos de potencia activa. Gao et al. (1994) diseñan un estabilizador no lineal de sistemas de potencia de estructura variable, mediante la técnica de transformación no lineal, la técnica de control de estructura variable y la teoría de sistemas lineales. El diseño logrado tiene la principal ventaja de que la sensibilidad ante variaciones en los parámetros del sistema, es notoriamente reducida. Lu et al. (1996), de nuevo usan la teoría geométrica diferencial, por medio de la linealización retroalimentada. En este caso, la ley de control obtenida presenta características valiosas por cuanto es óptima, descentralizada y no depende de los parámetros de la red de transporte. Zhu et al. (1997) contemplan la técnica de linealización retroalimentada en conjunto con la teoría de control robusto. En este caso, el diseño del controlador se efectúa para una máquina conectada a una red de potencia infinita, y su respuesta ante grandes perturbaciones presenta una considerable mejora en la regulación de tensión y la estabilidad transitoria. Wang et al. (1997) de nuevo, emplean la técnica de linealización retroalimentada en conjunto con la teoría de control robusto. Sin embargo, se generaliza la ley de control a un sistema multimáquina. Cao et al. (1998) diseñan un estabilizador de sistemas de potencia no lineal, utilizando la técnica de transformación retroalimentada no lineal y la regla de integración de la producción temporal y el error absoluto ITEA (integration of the production of time and absolute error). Mediante esta combinación, se logra que la ley de control sea descentralizada e insensible a los errores y variaciones en los parámetros del sistema. Finalmente, Akhrif \& Okou (1999) usan el esquema de linealización parcial retroalimentada. Esta técnica permite desacoplar las interacciones dinámicas en los distintos lazos de control, para estabilizar las oscilaciones del ángulo del rotor y mejorar la regulación de tensión de una máquina sincrónica.

Estas propuestas, sin embargo, presentan metodologías algo elaboradas o, en su defecto, la estructura obtenida en la ley de control es un tanto laboriosa de implementar físicamente. Por ambas razones, este artículo tiene como objetivo obtener una sencilla ley de control, fácilmente implementable, que mejore la estabilidad del sistema ante grandes perturbaciones.

En este artículo se aplica la conocida técnica de linealización entrada-salida (Isidori, 1995), al sistema de control de un compensador en serie controlado por tiristores o TCSC, cuyo formalismo elimina la dependencia no lineal del modelo, transformándolo en un sistema lineal equivalente. Una vez logrado el sistema equivalente, se optimiza la ley de control mediante el desarrollo del control óptimo lineal (LOC: linear optimal control), con el propósito de obtener un buen desempeño del controlador. Esta metodología hace que la respuesta del sistema de excitación ante una gran perturbación, presente buenas propiedades transitorias, cuando se compara con el desempeño de un sistema de control AVR/PSS estándar.

\section{TÉCNICA DE LINEALIZACIÓN ENTRADA-SALIDA (ISIDORI, 1995)}

Considere un sistema no lineal con una única señal de control de la forma: 
$\dot{x}=f(x)+g(x) \cdot u$

$y=h(x)$

donde $x \in R^{n}$ y $u \in R$ son las variables de estado y la variable de control, respectivamente, $y \in R$ denota la variable de salida, $f$ y $g$ son funciones vectoriales no lineales y $h$ es una función escalar de $x$.

Este sistema se dice que tiene un grado relativo $r$ en el punto $x_{o}$, si $r$ es el entero más pequeño tal que

1. $\quad L_{g} L_{f}^{i} h(x)=0$ para $i=0, \ldots, r-2$ para todo $x$ en la cercanía de $x_{o}$

2. $L_{g} L^{r-1} h(x) \neq 0$

donde $L_{f}$ y $L_{g}$ son las derivadas de Lie definidas como

$L_{f} h(x)=\sum_{i=1}^{n} \frac{\partial h(x)}{\partial x_{i}} f_{i}(x)$

$L_{g} h(x)=\sum_{i=1}^{n} \frac{\partial h(x)}{\partial x_{i}} g_{i}(x)$

Para establecer la forma normal de (1) en un nuevo sistema coordenado $\mathbf{Z}$, el mapeo coordenado debe seleccionarse de

$$
\mathbf{Z}=\left[\begin{array}{c}
h(x) \\
L_{f} h(x) \\
\vdots \\
L_{f}^{n-1} h(x)
\end{array}\right]
$$

Si el grado relativo $r$, es igual al orden del sistema $n$, la ecuación (1) puede transformarse a la siguiente forma normal,

$$
\begin{aligned}
& \dot{z}_{1}=z_{2} \\
& \dot{z}_{2}=z_{3} \\
& \cdots \\
& \dot{z}_{n-1}=z_{n} \\
& \dot{z}_{n}=\alpha(x)+\beta(x) \cdot u
\end{aligned}
$$

donde

$$
\begin{aligned}
& \alpha(x)=L_{f}^{n} h(x)=\frac{\partial\left(k_{f}^{n-1} h(x)\right)}{\partial x} f(x) \\
& \beta(x)=L_{g} L_{f}^{n-1} h(x)=\frac{\partial\left(k_{f}^{n-1} h(x)\right)}{\partial x} g(x)
\end{aligned}
$$

Para lograr que (4) sea un sistema lineal, debe asumir que

$\alpha(x)+\beta(x) \cdot u=v$

donde $v$ es el desempeño del nuevo controlador

Tomando en cuenta la suposición (6), el sistema (4) se transforma en la llamada forma normal de Brunovsky

$\dot{\mathbf{Z}}=\mathbf{A Z}+\mathbf{B V}$

donde

$\mathbf{Z}=\left[\begin{array}{c}z_{1} \\ z_{2} \\ \vdots \\ z_{n}\end{array}\right], \quad \mathbf{A}=\left[\begin{array}{cccccc}0 & 1 & 0 & \cdots & 0 & 0 \\ 0 & 0 & 1 & \cdots & 0 & 0 \\ \vdots & \vdots & \vdots & \vdots & \vdots & \vdots \\ 0 & 0 & 0 & \cdots & 0 & 1 \\ 0 & 0 & 0 & \cdots & 0 & 0\end{array}\right] \quad \mathrm{y} \quad \mathbf{B}=\left[\begin{array}{c}0 \\ 0 \\ \vdots \\ 0 \\ 1\end{array}\right]$

El sistema (7) además de ser linealizado es completamente controlable, mediante la ley de control

$u=-\frac{\alpha(x)}{\beta(x)}+\frac{1}{\beta(x)} v$

Ahora bien, como (7) es un sistema lineal, un buen desempeño del controlador $\mathbf{V}$ se logra mediante la técnica de diseño del control óptimo lineal, tal como el método LQR. El método LQR optimiza el índice de desempeño cuadrático

$$
J=\frac{1}{2} \int_{0}^{\infty}\left(\mathbf{Z}^{\mathrm{T}} \mathbf{Q Z}+\mathbf{V}^{\mathrm{T}} \mathbf{R} \mathbf{V}\right) \cdot d t
$$


minimizando el gasto de energía del sistema de control a través de la matriz de peso semidefinida positiva $\mathbf{Q}$ de dimensión $n x n$, y mediante la matriz de peso definida positiva $\mathbf{R}$ de dimensión $m \times m$ ( $m$ es el número de señales de control).

Con esta metodología se tiene que la máxima eficiencia del controlador se alcanza cuando

$\mathbf{V}^{*}=-\mathbf{K}^{*} \mathbf{Z}$

donde $\mathbf{V}^{*}$ denota un vector de control óptimo y $\mathbf{K}^{*}$ es la ganancia óptima retroalimentada, dada por

$\mathbf{K}^{*}=\mathbf{R}^{-1} \mathbf{B}^{\mathbf{T}} \mathbf{P}^{*}$

siendo $\mathbf{P} *$ la solución de la ecuación algebraica de Riccati

$$
\mathbf{A}^{\mathrm{T}} \mathbf{P}+\mathbf{P A}-\mathbf{P B R} \mathbf{R}^{-\mathbf{1}} \mathbf{B}^{\mathrm{T}} \mathbf{P}+\mathbf{Q}=\mathbf{0}
$$

\section{MODELO DINÁMICO Y LEY DE CONTROL DEL SISTEMA.}

\section{A. Modelo dinámico}

En esta sección, el diseño de un control no lineal de un TCSC es ilustrado usando el sistema de la Figura. 1. El sistema de prueba consiste de dos sistemas de potencia vinculados mediante una línea de transmisión, en el cual la potencia activa se transporta del sistema "i" al sistema "j".
En este modelo, cada área representa un grupo de máquinas sincrónicas que se mantienen en operación coherente. El modelo dinámico de cada grupo de máquinas consiste en un sistema de segundo orden, por cuanto se quiere analizar la respuesta de las variables eléctricas en el vínculo que las une. Se asume que la dinámica del sistema, antes de la perturbación, se modela mediante la potencia activa en régimen permanente $P_{\text {eijo }}$. Con esta simplificación, las ecuaciones del sistema son

$\left[\begin{array}{l}\dot{\delta}_{i j} \\ \dot{\omega}_{i j}\end{array}\right]=\left[\begin{array}{l}\omega_{i j}-\omega_{i j o} \\ \frac{\omega_{i j o}}{2 H_{i}} P_{e i j o}\end{array}\right]+\left[\begin{array}{c}0 \\ -\frac{\omega_{i j o}}{2 H_{i}} V_{i} V_{j} \cdot \operatorname{sen} \delta_{i j}\end{array}\right] \cdot u$

donde

$u=x_{12}^{-1}, \quad x_{12}=x_{L}-x_{T C S C}, x_{L}=x_{1}+x_{2}$

\section{B. Transformación a un sistema lineal equivalente}

La ecuación (13) puede interpretarse en la teoría de sistemas dinámicos como un sistema no lineal afín

$\dot{x}=f(x)+g(x) \cdot u$

donde $x=\left[\delta_{\mathrm{ij}} \omega_{\mathrm{ij}}\right]^{\tau}$ es el vector de estado y $u$ es la variable de control del TCSC.

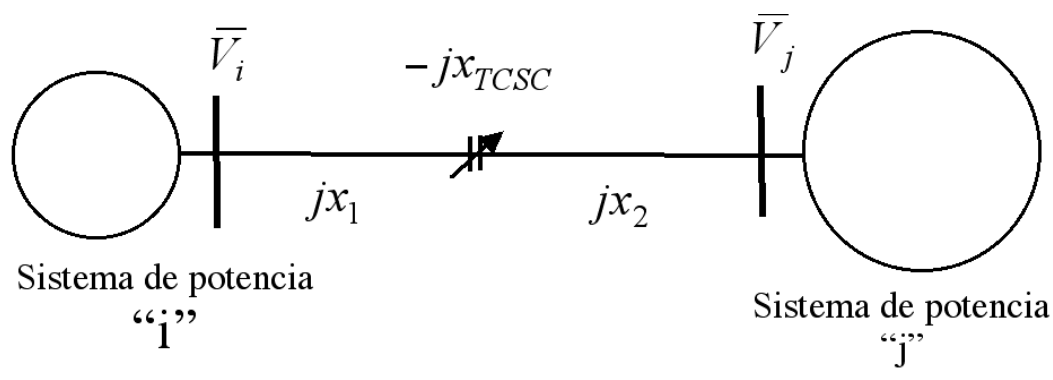

Figura 1. Modelo del sistema de dos áreas. 
Puesto que el objetivo del diseño propuesto es mejorar la estabilidad del sistema ante una gran perturbación, se utiliza la desviación en el ángulo de potencia $\delta_{i j}$ como la ecuación de salida del controlador. Definiendo la salida $y$ como

$y=h(x)=\delta_{i j}-\delta_{i j o}$

Una vez establecida la estructura de (1), y de acuerdo con la sección 3, el siguiente paso es calcular las derivadas de Lie, las cuales son

$$
\begin{aligned}
& L_{g} L_{f}^{o} h(x)=L_{g} h(x)=0 \\
& L_{f} h(x)=\omega-\omega_{o} \\
& L_{g} L_{f} h(x)=-\frac{\omega_{i j o}}{2 H_{i}} V_{i} V_{j} \operatorname{sen} \delta_{i j}
\end{aligned}
$$

Si en (18) $\sin \delta \neq 0$ es diferente de cero, lo cual es una condición de operación factible, se concluye que el grado relativo del sistema es 2, y es igual al orden de (13), de modo que se puede emplear el método propuesto en la sección 2 para el diseño de un TCSC no lineal.

A partir de (3), la transformación de coordenadas al nuevo sistema es:

$\ddot{O}(x)=Z=\left[\begin{array}{c}\ddot{a}_{i j}-\ddot{a}_{i j o} \\ \grave{u}_{i j}-\grave{u}_{i j o}\end{array}\right]$

Para comprobar que (19) da origen a un sistema lineal y a la vez controlable, una condición necesaria y suficiente es que el determinante de la Matriz Jacobiana de $\Phi(\mathrm{x})$ sea diferente de cero (Isidori, 1995). La Matriz Jacobiana de $\Phi(\mathrm{x})$ es,

$J_{\Phi}=\frac{\partial \Phi(x)}{\partial x}=\left[\begin{array}{ll}1 & 0 \\ 0 & 1\end{array}\right]$

siendo su determinante

$$
\operatorname{det}\left(J_{\Phi}\right)=1
$$

Si $\sin \delta_{i j} \uparrow 0$, lo cual es una condición de operación real, el sistema no lineal (13) puede transformarse a través de (19), en un sistema lineal y controlable en el subconjunto

$\Omega=\left\{\delta_{i j}, \quad \omega_{i j} \mid \operatorname{sen} \delta_{i j} \neq 0\right\}$

Por tanto, el sistema lineal resultante es

$$
\begin{aligned}
{\left[\begin{array}{l}
\dot{z}_{1} \\
\dot{z}_{2}
\end{array}\right] } & =\left[\begin{array}{ll}
0 & 1 \\
0 & 0
\end{array}\right]\left[\begin{array}{l}
z_{1} \\
z_{2}
\end{array}\right]+\left[\begin{array}{l}
0 \\
1
\end{array}\right] v \\
y & =z_{1}
\end{aligned}
$$

\section{Diseño de la ley de control no lineal}

Sustituyendo (13) y (19) en (23), se tiene que

$v=\frac{\omega_{i j o}}{2 H_{i}} P_{e i j o}-\frac{\omega_{i j o}}{2 H_{i}} V_{i} V_{j} \cdot \operatorname{sen} \delta_{i j} \cdot u$

siendo la ley de control

$u=\frac{P_{\text {eijo }}-\frac{2 H_{i}}{\omega_{i j o}} v}{V_{i} V_{j} \sin \delta_{i j}}$

Ahora bien, para mejorar la eficiencia de (25) se buscará una entrada óptima de $v^{*}$, mediante la ecuación algebraica de Riccati. A partir de (23), se conocen las matrices A y $\mathbf{B}$ de (7).

Como sólo hay un dispositivo de control, la matriz $\mathbf{R}$ es un escalar cuyo valor se mantiene como una incógnita. Además, asumiendo que $z_{1}$ es la única variable que participa en la dinámica del sistema, se selecciona $\mathbf{Q}=\operatorname{diag}\left(\begin{array}{ll}1 & 0\end{array}\right)$ en la ecuación de Riccati.

De esta forma, la ley de control óptimo es

$$
x_{T C S C}=x_{L}-\frac{V_{i} V_{j} \sin \delta_{i j}}{P_{e i j o}+\frac{2 H_{i}}{\omega_{i j o}}\left(k_{1} \Delta \delta_{i j}+\sqrt{2 k_{1}} \Delta \omega_{i j}\right)}
$$


Los autovalores $\lambda$ y el amortiguamiento relativo del sistema lineal para cualquier valor de $k_{l}$ son

$\lambda=-\sqrt{\frac{k_{1}}{2}} \pm j \sqrt{\frac{k_{1}}{2}}$

$\xi=70,7 \%$

Sin embargo, la reactancia $x_{T C S C}$ esta limitada por los límites de operación del TCSC. De modo tal, que el valor de $k_{l}$ debe ser seleccionado para adaptarse a los cambios de potencia transportada y reducir el efecto de las limitaciones de operación del TCSC. Con base en las limitaciones de operación del TCSC, la ley de control debe satisfacer la siguiente condición:

$k_{1} \Delta \delta_{i j}+\sqrt{2 k_{1}} \Delta \omega_{i j}-\frac{\omega_{i j o}}{2 H_{i}} \Delta P_{e i j}=0$

De esta forma, para cualquier transferencia de potencia, el valor de $k_{l}$ puede calcularse fácilmente mediante técnicas iterativas, tales como Gauss-Seidel o Newton Raphson.

Es importante enfatizar que en (26) todos los parámetros del sistema pueden medirse directamente. Así, de la potencia activa y reactiva en el extremo receptor " $j$ " de la línea, se tiene que

$\delta_{i j}=\arctan \left\{\frac{P_{e j}\left(x_{L}-x_{T C S C}\right)}{Q_{e j}\left(x_{L}-x_{T C S C}\right)+V_{j}^{2}}\right\}$

$\omega_{i j}=\dot{\delta}_{i j}$

De las relaciones anteriores, con el TCSC localizado en la línea de enlace, no es posible proporcionar amortiguamiento a todos los modos de oscilación. El estabilizador de sistemas de potencia puede utilizarse para resolver las oscilaciones de tipo local (Chapman et al., 1993), pero, este tópico está fuera del alcance de este artículo.

\section{SIMULACIÓN DEL SISTEMA}

A continuación se presentan las simulaciones efectuadas al sistema de la Figura 1. Los parámetros del sistema y las condiciones iniciales de operación, se muestran en el apéndice. La simulaciones fueron efectuadas usando el Mathworks "Power System" Blockset (Mathworks, 1999).

Para evaluar el desempeño del controlador no lineal, el sistema fue sometido a una falla trifásica simétrica en el extremo receptor de la línea de transmisión. La falla se inicia a los 0,1 $\mathrm{s}$ y se libera a los $0,2 \mathrm{~s}$. La respuesta del control no lineal se compara con el diseño clásico de un capacitor en serie de valor constante.

A partir de la Figura 2 se observa cómo el control no lineal mejora la respuesta del sistema de control del TCSC, logrando que la respuesta de potencia activa a través de la línea se estabilice después de los 0.2 segundos, después de restablecida la línea de transmisión.

La Figura 3 muestra cómo el desempeño del control no lineal es similar al diseño tradicional de compensación fija. Ante esta situación, la desviación de la tensión recupera su condición prefalla en una manera suave, después del concebido transitorio de conmutación, sin generar ciertas oscilaciones, como sí sucede en los otros sistemas de control. Note que estas oscilaciones repercuten en la regulación de tensión del sistema.

De la Figura 4 se desprende cómo el control no lineal nuevamente logra que la corriente, a través de la línea de transmisión, alcance la nueva condición de régimen permanente de una forma suave. El desempeño del control no lineal, hace que la corriente se estabilice en menos de 0,3 segundos, después de eliminada la contingencia. 


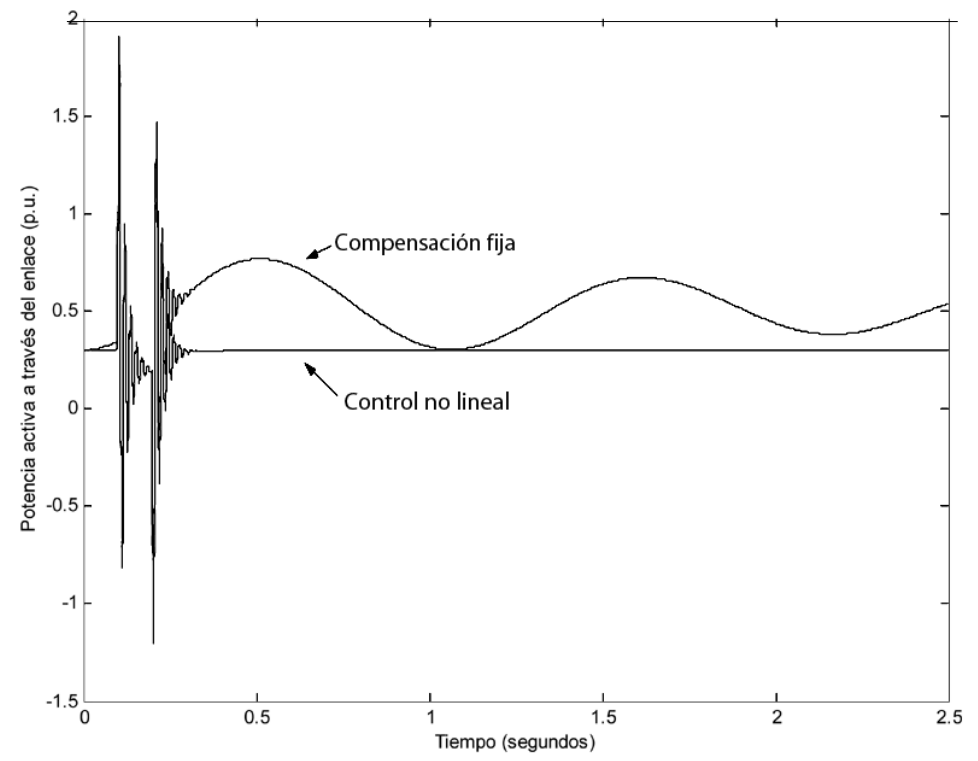

Figura 2. Desempeño del sistema de control del TCSC ante la falla trifásica.

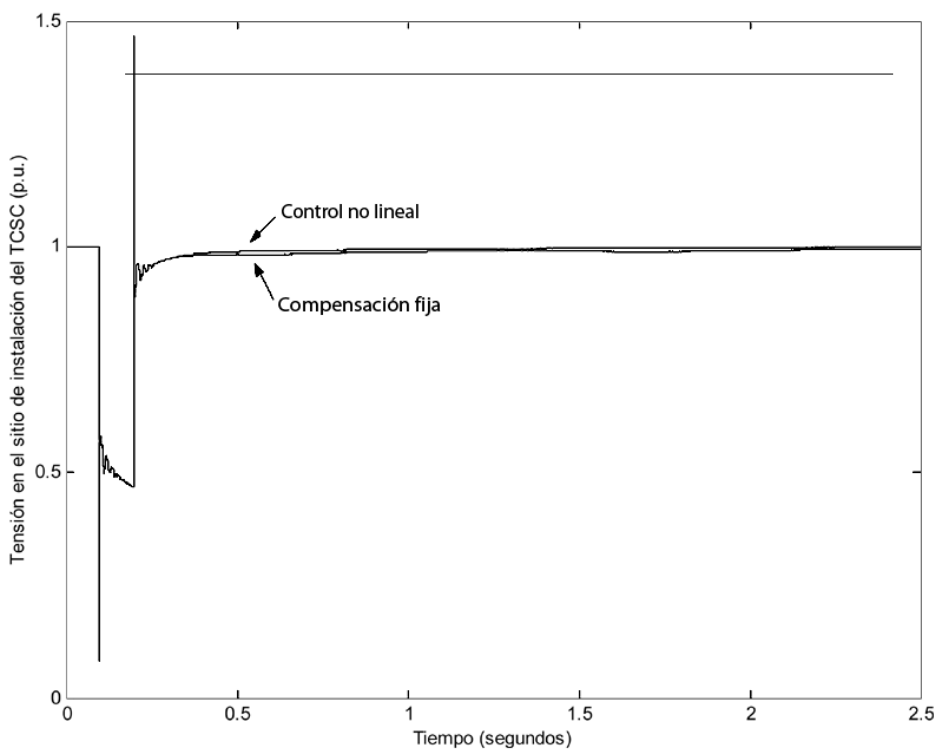

Figura 3. Respuesta de la tensión en sitio de instalación del TCSC. 


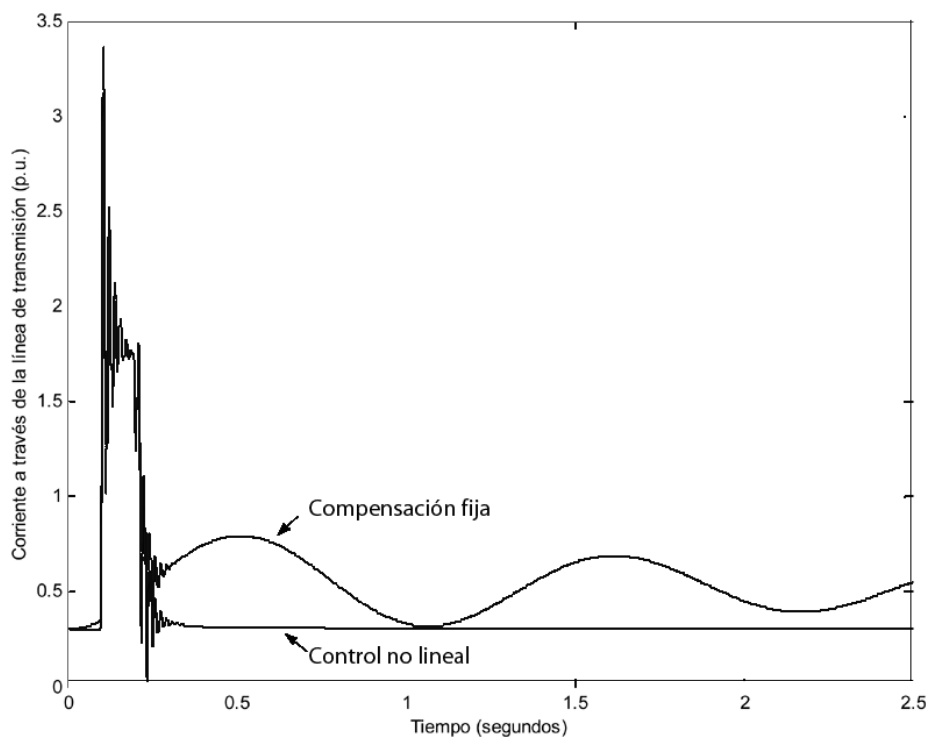

Figura 4. Variación de la corriente a través de la línea de transmisión.

\section{CONCLUSIONES}

De los resultados obtenidos se desprenden las siguientes conclusiones:

1. El control no lineal propuesto presenta una respuesta satisfactoria en todas las variables de interés, y supera al desempeño obtenido por los diseños convencionales de compensación fija.

2. La ley de control obtenida mediante la técnica entrada-salida, permite que todos los parámetros involucrados puedan medirse directamente.

3. Puesto que la ley de control (26) depende del punto de operación, su desempeño será robusto.

\section{SIMBOLOGÍA}

$\delta_{i j}$ : $\quad$ desfase entre la barra "i” y la barra “j”, radianes

$\omega_{i j} \quad$ velocidad angular entre la barra "i" y la barra "j", radianes/s

$H_{i} \quad$ constante de inercia en la barra "i", $\mathrm{s}$

$P_{e i j} \quad$ potencia active de la barra "i" a la barra "j", p.u.

$P_{e j} \quad$ potencia active que llega a la barra “j”, p.u.

$Q_{e j}: \quad$ potencia reactive que llega a la barra “j”, p.u.

$k_{1}$ : ganancia óptima retroalimentada del TCSC, p.u.

$u$ : $\quad$ variable de control del TCSC, p.u.

$x_{T C S C}$ : reactancia capacitiva del TCSC, p.u.

$x_{L}: \quad$ reactancia de la línea de transmisión, p.u. 
$\bar{V}_{i}: \quad$ fasor de tensión en la barra "i", p.u.

$\bar{V}_{j}$ : fasor de tensión en la barra “j”, p.u.

$o$ : subíndice que indica la condición en régimen permanente

\section{APÉNDICE}

Los parámetros del sistema están en p.u. sobre una base de 100 MVA,

\begin{tabular}{|c|c|c|c|}
\hline$H_{i}=8,5 \cdot s$ & $V_{i}=1,0$ & $V_{j}=1,0$ & $k_{1}=1$ \\
\hline$\delta_{i j o}=17,45^{\circ}$ & $x_{L}=1,0$ & $P_{\text {eijo }}=0,3$ & $\omega_{i j o}=1,0$ \\
\hline
\end{tabular}

\section{BIBLIOGRAFÍA}

Akhrif, O., Okou, F. A., Dessaint, L. A., Champagne, R. (1999, May.). Application of a multivariable feedback linearization scheme for rotor angle stability and voltage regulation of power systems, IEEE Trans. Power Systems, 2(14),620-628.

Cao, Y. J., Wu, Q. H., Jiang, L., Cheng, S. J. (1998). Nonlinear control of power systems multi-mode oscillations, Electrical power \& Energy Systems, 1(20),61-68.

Chapman, J. W., Ilic, M. D., King, C. A., Eng, L., Kaufman, H. (1993, Aug.). Stabilizing a multimachine power system via decentralized feedback linearizing excitation control, IEEE Trans. Power Systems, 3(8),830-839.

Gao, Y., Jiang, L., Cheng, S., Chen, D., Malik, O. P., G. S. (1994, Sept.). A nonlinear variable structure stabilizer for power system stability, IEEE Trans. Energy Conversion, 3(9),489-495.
Isidori, A. (1995). Nonlinear Control System: An Introductions. Berlin: Springer-Verlag, 137-147.

Kundur, P. (1994). Power Systems Stability and Control. New York: McGraw-Hill, 766-781.

Lu, Q. \& Sun, Y. Z. (1989, Feb.). Nonlinear stabilizing control of multimachine systems, IEEE Trans. Power Systems, 1(4), 236-241.

Lu, Q., Sun, Y., Xu, Z., Mochizuki, T. (1996, Nov.). Decentralized nonlinear optimal excitation control, IEEE Trans. Power Systems, 4(11),1957-1962.

The Mathworks (1999, Jan.). Power System Blockset-User's guide.

Wang, Y., Guo, G., Hill, D. J. (1997). Robust decentralized nonlinear control design for multimachine power systems, Automatica, 9(33),1725-1733.

Zhu, C., Zhou, R., Wang, Y. (1997). A new nonlinear voltage controller for power systems, Electrical Power \& Energy Systems, 1(19),19-27.

\section{SOBRE EL AUTOR}

\section{Eddie A. Araya Padilla}

Ingeniero electricista, $\mathrm{Ph}, \mathrm{D}$.; profesor del Departamento de Sistemas de Potencia de la Escuela de Ingeniería Eléctrica de la Universidad de Costa Rica

Teléfonos: 207-4329 o 207-5146

Facsímil: 207-4139

Correo electrónico: earaya@eie.ucr.ac.cr 